\title{
Changing the Culture of Tumor Tissue Biobanking in a Tertiary Referral Hospital Using an Audit and Feedback Strategy
}

\author{
Simon King,, ${ }^{1,2}$ Marjorie Walker, ${ }^{1-3}$ Sarah Neilsen, ${ }^{2}$ and Michelle O'Brien ${ }^{1}$
}

We examined whether the introduction of an audit and feedback strategy applied to specimens dissected at the John Hunter Hospital anatomical pathology department improved the number of formalin fixed tumor specimens sampled by anatomical pathology registrars for the Hunter Cancer Biobank. During the audit period (1/7/16 to 30/6/17) a total of 949 tumor specimens were sampled for the biobank compared with 393 of the previous year (1/7/15 to 30/6/ 16) resulting in a $141 \%$ increase in specimens biobanked. A targeted group of previously underrepresented specimen types, including brain, lung, and lymph node tumors were studied in depth to establish which specimens were sampled and which potentially biobankable specimens were "missed". In this targeted group there was a $285 \%$ increase in the number of specimens biobanked and a statistically significant $(p<0.001)$ increase in tumor sampling in all three specimen types over the audit period compared with the previous year. In conclusion, the introduction of an audit and feedback strategy improved tumor tissue collection for biobanking. A potential drawback of email feedback is that overfixation may occur due to administrative time lag and these specimens must be documented accordingly because some tests are dependent on an optimal fixation time. Taking extra blocks for biobanking on all potential tumor specimens with excess tumor tissue at the time of cut-up can alleviate this problem.

Keywords: biobanking, formalin, human

\section{Introduction}

$\mathrm{T}$ HE OECD (ORganisation for Economic Co-operation and Development) defines a biobank as a "collection of biological material and the associated data and information stored in an organized system, for a population or a large subset of a population." "Human tissue biobanking is vital to modern medical research, evolving our knowledge of health and disease by increasing the availability of tumor tissue to advance translational cancer research in this era of personalized medicine.

The Hunter Cancer Biobank (HCB) works closely with the anatomical pathology department at the John Hunter Hospital and Hunter New England local health district to collect, process, and store formalin fixed paraffin embedded (FFPE) cancer biospecimens, allowing them to be paired with appropriate clinical, immunohistochemical, and molecular data from local clinical information systems, which can then be accessed by scientists and clinicians involved in research.
Anatomical pathology is at the heart of the biobanking process and a close relationship between a tumor biobank and its allied anatomical pathology department is essential to maximize the number and variety of cancer biospecimens available to scientific and clinical researchers both locally and interstate.

The John Hunter Hospital Anatomical Pathology laboratory is one of the largest in New South Wales, processing over 34,000 specimens per annum. Biobanking is performed by anatomical pathology registrars and histology technicians at the time of macroscopic description and specimen dissection, but it is only performed if excess tumor is available after routine diagnostic sampling has taken place.

Up until 2015 the HCB held 12,000 blocks of tissue from 4500 patients. However, on closer assessment only $20 \%$ of cancer resection specimens (complexity $6-7^{2}$ ) received by the John Hunter Hospital anatomical pathology department were sampled, the majority of which were taken from breast and colorectal specimens, whereas other specimens such as brain,

${ }^{1}$ Department of Anatomical Pathology, John Hunter Hospital, Newcastle, NSW, Australia.

${ }^{2}$ Hunter Cancer Biobank, Hunter Medical Research Institute (HMRI), New Lambton Heights, NSW, Australia.

${ }^{3}$ Department of Medicine, University of Newcastle, Callaghan NSW, Australia.

(C) Simon King et al., 2019; Published by Mary Ann Liebert, Inc. This Open Access article is distributed under the terms of the Creative Commons Attribution Noncommercial License (http://creativecommons.org/licenses/by-nc/4.0/) which permits any noncommercial use, distribution, and reproduction in any medium, provided the original author(s) and the source are cited. 
lung, and lymph node tumors represented a comparatively small proportion of tumor resection specimens sampled, constituting $3 \%, 5 \%$, and $2 \%$ respectively.

These statistics would suggest that we are not maximizing the biobanking potential of all tumor resection specimens received by the anatomical pathology department.

The aim of this study was to determine whether the implementation of a prospective audit and feedback strategy within the anatomical pathology department would increase the number of formalin fixed tumor specimens sampled (biobanked) for the HCB.

\section{Methods}

Ethical approval for biobanking and auditing of the data was given by the Hunter New England Human Research Ethics Committee (HNEHREC 12/06/20/S.03).

\section{Outline of tumor biobanking within the anatomical pathology department}

In our department tumor biobanking is undertaken during the macroscopic assessment when excess tumor is available (Fig. 1). The tumor blocks for biobanking are taken at the same time as the diagnostic blocks and submitted in specially designated, light gray tissue cassettes that are then processed alongside the diagnostic blocks. Biobanking blocks are then separated into a box for collection by a biobank technician and taken to the biobank where they are registered on a database (Openspecimen), paired with clinical and pathological data and then stored in a temperature controlled, low light environment.

Before July 1, 2016, every new intake of anatomical pathology registrars was given guidance on the importance of biobanking tumor samples from suitable specimens with ad hoc reminders during the year.

From July 1, 2016 to June 30, 2017, an audit and feedback strategy was initiated in addition to usual guidance with the aim to increase the number of tumor specimens sampled for the HCB.

\section{Outline of the audit and feedback strategy applied to tissue biobanking}

The aim of the audit and feedback process in our study is to assess which specimens received by the anatomical pathology department should have been biobanked at the time of specimen dissection (Fig. 2).

To perform this task a biobank technician performs a weekly search using the laboratory information system (LIS), using a specified date range and range of specimen complexity.

The database search is restricted to complexity levels 5 to $7,{ }^{2}$ because this complexity range encompasses most tumor specimens providing the majority of tumors for biobanking.

A table of anatomical pathology specimens matching these criteria is then generated with accompanying information including the laboratory number, date of receipt, and specimen type.

Tumor specimens with blocks received by the biobank are then removed from this list, resulting in a list of patient specimens that have not been sampled. Non-tumor resection specimens such as LETTZ biopsies, medical renal biopsies, and benign thyroid specimens that attract complexity 5 or above are then removed together with pediatric specimens, which are not covered by our ethics waiver.

This list of potentially biobankable tumor specimens is then sent to the "biobank pathologist" who assesses each specimen's pathology report to see whether there is a reason that a tumor sample could not be taken, such as small tumor size or if the lesion removed is benign.

The biobank pathologist then sends a "feedback" email requesting tumor tissue for biobanking to the anatomical pathology registrar responsible for dissecting the specimen. Once they go back to the specimen, they record whether or not tumor sampling was performed in the "specimen notes" section of the LIS.

For the first part of this study we compared the total number of tumor specimens biobanked under the audit and feedback strategy with the same period the year before.

For the second part of the study we critically evaluated the impact of an audit and feedback strategy on tumor biobanking by focusing on three previously underrepresented specimen types in depth, chosen based on their low numbers relative to the number of specimens received in the department. The specimens chosen included brain, lymph node, and lung tumor resection specimens. These specimen types were retrospectively evaluated in detail between $1 \mathrm{st}$ July 2015 to 30 June 2016 and during the audit period from 1st July 2016 to 30th June 2017 to determine whether tumors deemed to be eligible for biobanking based on their macroscopic reports had been sampled.

\section{Statistical methods employed}

The number of brain, lymph node, and lung tumor resection specimens biobanked between 2017 and 2016 were compared to those during 2016 to 2015 using $2 \times 2$ contingency tables. $p$ values for the difference in data recorded between the two groups were generated using the chisquared statistical test.

\section{Results}

Figure 3 summarizes the results of the audit and feedback strategy on the number of specimens and specimen types sampled for the HCB by the anatomical pathology department during the audit and feedback period (1st July 2016 to 30th June 2017) compared to the same period over previous years.

All specimen types demonstrated an increase in the number of specimens biobanked during the audit period. In total, 949 individual tumor specimens were sampled for the biobank between 1st July 2016 and 30th June 2017 compared with 393 in the previous period, representing a $141 \%$ increase.

Although all specimen types saw large increases in the number of tumor specimens sampled over the audit period, the largest increases were seen in specimens previously underrepresented when comparing biobank data from previous years with specimens received by anatomical pathology department including brain, lung, and lymph node specimens (Fig. 3).

The specimens targeted in depth all saw large increases in the number of specimens sampled, with lymph node and brain specimens seeing an increase of greater than $285 \%$ during the audit period and lung tumor sampling more than doubled during this time. 
FIG. 1. Flowchart illustrating the process of specimen handling and tissue biobanking within the anatomical pathology department.

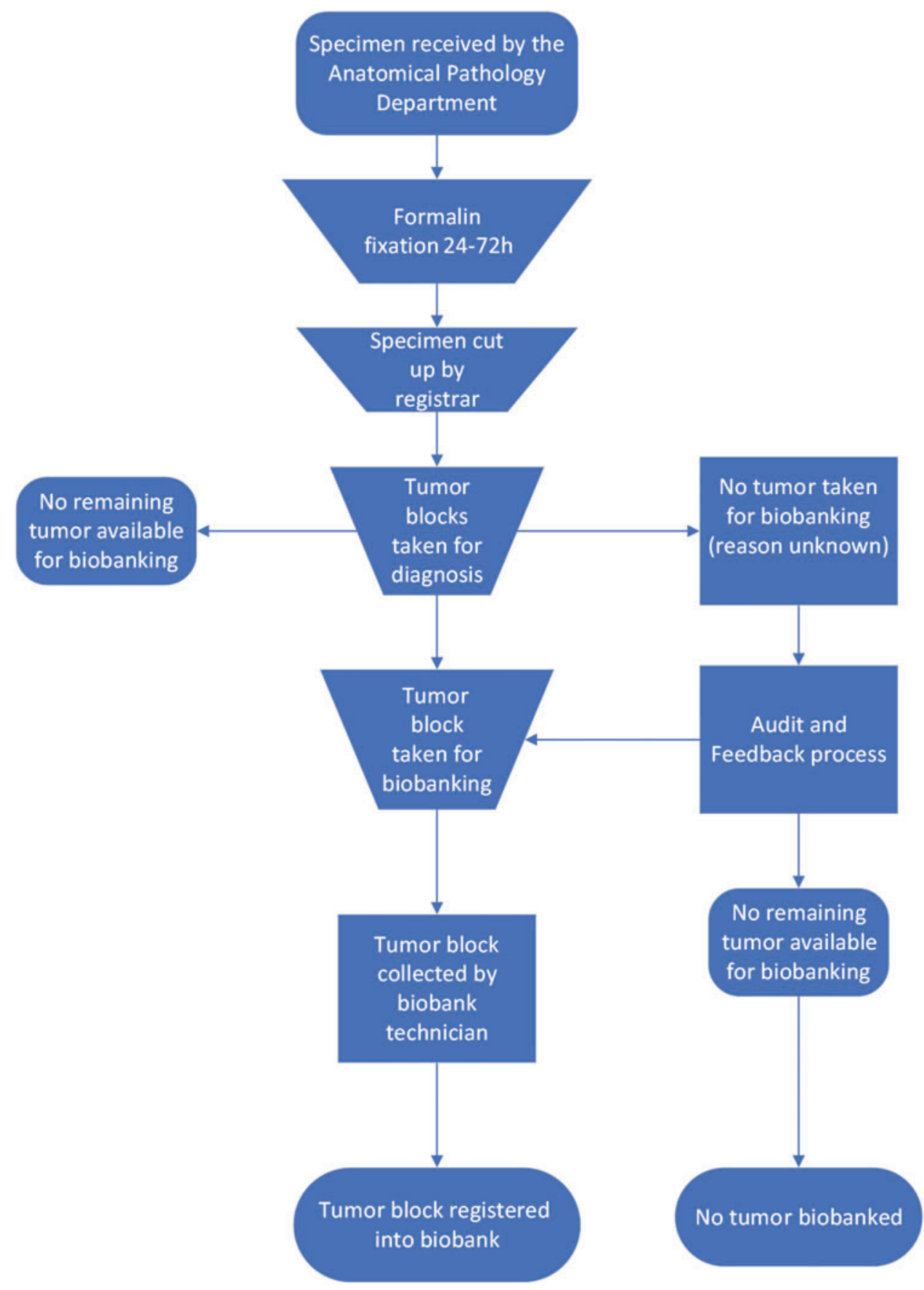

During the audit and feedback strategy a total of $59(88 \%)$ brain tumors were sampled out of a potential 67 cases deemed to be amenable to biobanking. This compares to only $16(25 \%)$ of specimens biobanked out of a possible 65 specimens between 1st July 2015 and 30 June 2016. Out of the 67 brain tumor cases amenable to biobanking between 2016 and 2017, 19 cases were not sampled at initial specimen dissection. Of these 19 potentially biobankable cases 17 feedback emails were sent and in the remaining 2 cases no email was sent due to our administration error.

Of the 17 feedback emails sent, 11 specimens were subsequently sampled and in 6 cases no response or subsequent tumor block was received from the registrar responsible.

In the case of lung tumors, $43(93 \%)$ out of a potential 46 specimens were sampled during the audit period compared to $11(30 \%)$ from 37 specimens in the preceding period. Out of the 46 cases amenable to biobanking, 39 (85\%) of cases were sampled at initial dissection with only 7 potentially biobankable cases not sampled, prompting 7 feedback emails, 4 of which generated tumor samples but 3 did not elicit a response or tumor banking block from the email recipient.

For lymph node excisions, $29(73 \%)$ out of a possible 40 were sampled compared with $7(13 \%)$ out of 56 specimens received in the preceding period. Out of the 40 cases assessed as amenable to biobanking $22(55 \%)$ were not sampled at initial specimen dissection. Of these 22 cases, 15 feedback emails were sent requesting biobanking, but in the remaining 7 cases no email was sent due to an administrative error.

Of the 15 feedback emails that were sent, 11 specimens were subsequently sampled, but in the remaining 4 cases no 


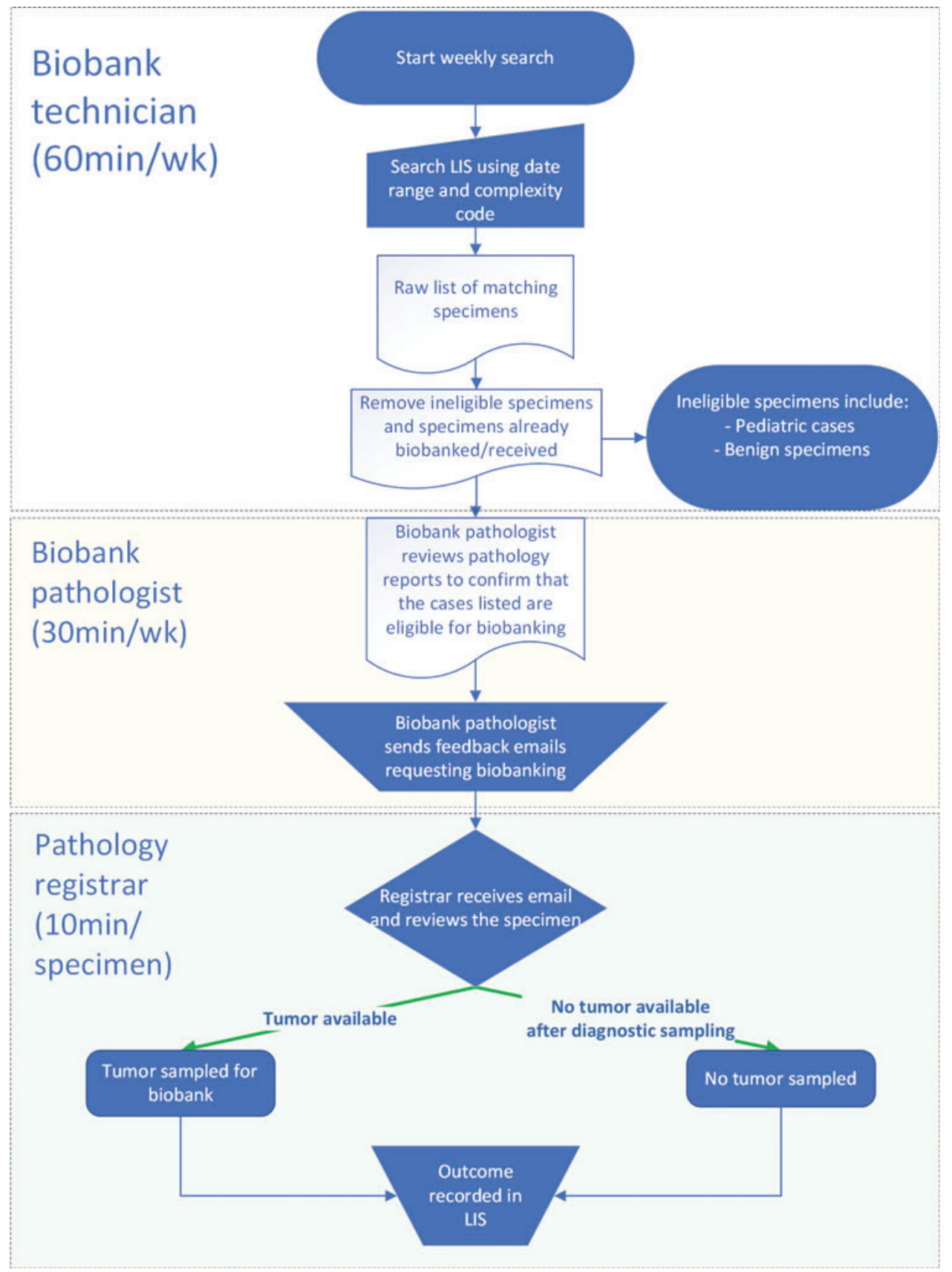

FIG. 2. Flowchart illustrating the audit and feedback process as applied to human tissue biobanking in a public hospital together with the time taken for each stage.

tumor sample or response from the registrar was received. All three specimen types audited in detail demonstrated a statistically significant $(p<0.0001)$ increase in the number of specimens sampled for biobanking during the audit period compared to the same period the preceding year using the Chi squared statistical test (Table 1).

Tumor sampling taken at the time of initial specimen dissection was also compared to tumor sampling after email feedback during the audit strategy. This revealed that email feedback significantly increased the number of lymph node and brain tumor specimens biobanked by $28 \%$ and $60 \%$ respectively compared to those sampled at initial dissection. No significant increase was seen in lung tumor resections because only a small number of cases were missed on initial dissection, highlighting the success an audit and feedback strategy has on improving tumor sampling at initial specimen dissection.

\section{Discussion}

Over the past 30 years tissue biobanks have become important resources in medical research, supporting contemporary areas of research such as genetics, genomics, and proteomics, and while traditionally DNA and RNA analyses have been performed on fresh tissue, advances in DNA and RNA extraction techniques have enabled the use of archival FFPE material to provide DNA and RNA of comparable quality for molecular testing. ${ }^{3-5}$

To improve on this resource, we employed an audit and feedback strategy with a view to maximizing the number 
250

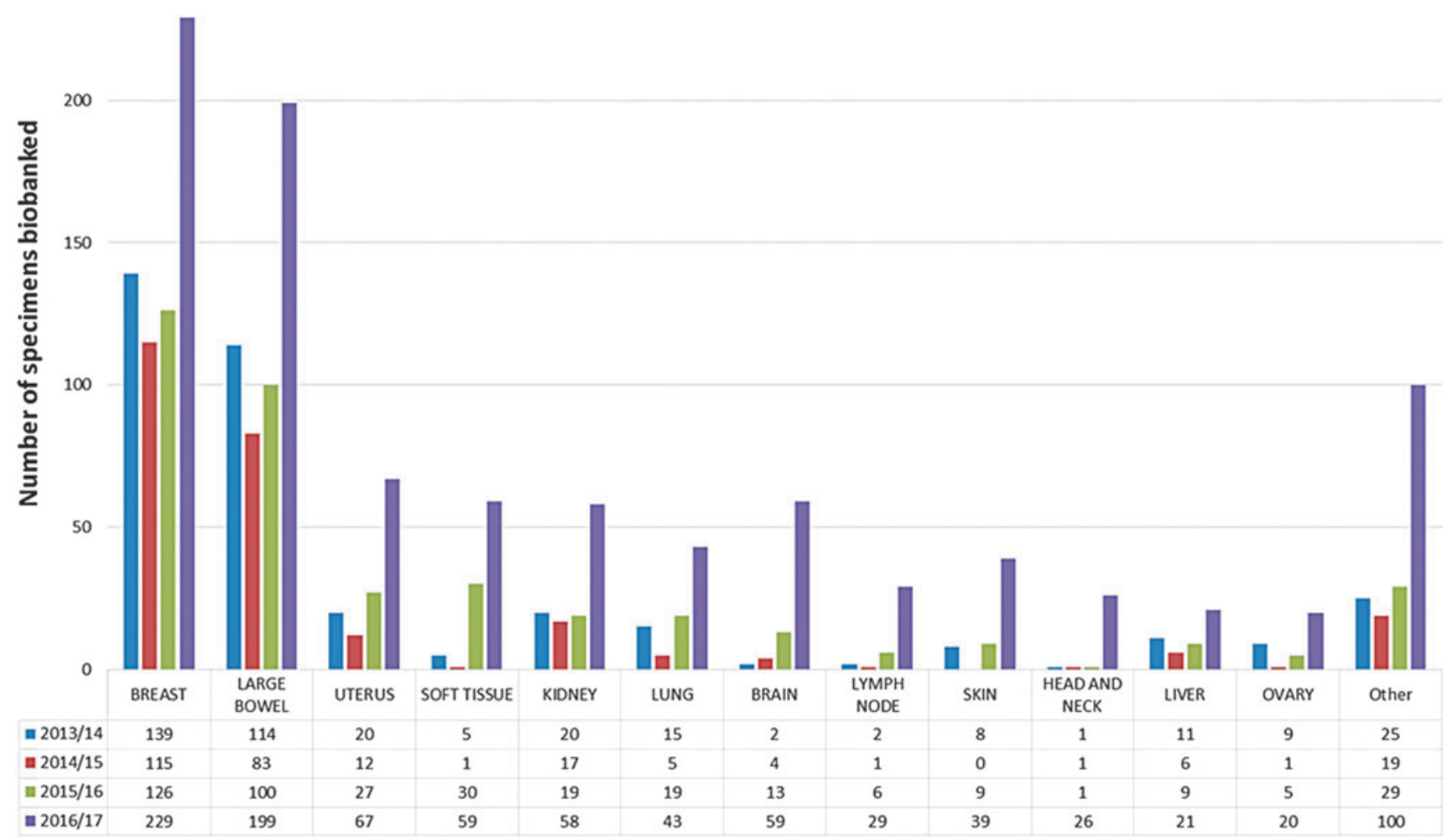

FIG. 3. Tumor specimens sampled for the Hunter Cancer Biobank by the anatomical pathology department at the John Hunter Hospital over a 5-year period.

and variety of tumor specimens biobanked from the anatomical pathology department. To the best of our knowledge this is the first study to have compared the impact of an audit and feedback strategy on the biobanking process by modifying the sampling behavior of anatomical pathology registrars. Over the last 20 years an audit and feedback strategy has been successfully used to modify professional behavior in many areas of medicine and is defined as "any summary of clinical performance of health care over a specified period of time," which allows them to adjust or modify their performance. ${ }^{6}$ The basis of this strategy is to monitor an individual's professional practice or performance that is then compared to expected standards or targets. The efficacy of this strategy can be highly variable and several studies have shown that audit and feedback is most effective when three criteria are met. ${ }^{7}$ First, baseline compliance with recommended practice must be low. Second, feedback should be given in writing by a supervisor, and finally the audit must include clear targets and a simple action plan.

We believe that our study design meets these criteria. For example, the current baseline performance of sampling tumor tissue for the biobank is low, with only $20 \%$ of tumor resection specimens being sampled. Second, feedback is given to anatomical pathology registrars by a supervisor

Table 1. Statistical Comparison of Specimen Types Studied in Depth with $P$-Values Comparing the Year 2016/2017 with 2015/2016 ( $P$-Value A) and Specimens Sampled Before ANd After Email FeedBack ( $P$-Value B)

\begin{tabular}{|c|c|c|c|c|c|c|c|}
\hline & \multicolumn{2}{|c|}{ Initial sampling } & \multirow[b]{2}{*}{ Total specimens } & \multirow[b]{2}{*}{$\mathrm{p}$-Value $(A)$} & \multicolumn{2}{|c|}{ After feedback email } & \multirow[b]{2}{*}{$\mathrm{p}$-Value $(B)$} \\
\hline & Sampled & Not sampled & & & Sampled & Not sampled & \\
\hline \multicolumn{8}{|l|}{ Brain } \\
\hline $2016 / 2017$ & 48 & 19 & 67 & $<0.0001$ & 59 & 8 & 0.018 \\
\hline $2015 / 2016$ & 16 & 49 & 65 & & & & \\
\hline \multicolumn{8}{|l|}{ Lung } \\
\hline $2016 / 2017$ & 39 & 7 & 46 & $<0.0001$ & 43 & 3 & 0.193 \\
\hline $2015 / 2016$ & 11 & 26 & 37 & & & & \\
\hline \multicolumn{8}{|l|}{ Lymph } \\
\hline $2016 / 2017$ & 18 & 22 & 40 & 0.00035 & 29 & 11 & 0.0125 \\
\hline $2015 / 2016$ & 7 & 49 & 56 & & & & \\
\hline
\end{tabular}


(anatomical pathologist) in a timely manner, (weekly) and third, the target is to maximize the number of tumor resection specimens received that are biobanked by the anatomical pathology department.

This belief is borne out in the results, with tumor blocks from 978 individual patient specimens representing a $141 \%$ increase over the previous year.

Moreover, we have also seen an increase in tumor banking across all the specimen types collected, with the largest increases seen in tumor specimens that have been previously underrepresented in the biobank, such as kidney and uterus in addition to the three targeted specimens (Fig. 3). The number of breast and colorectal tumor specimens sampled have also increased, but collectively their overall proportion has dropped when compared with the previous year, due to the larger increases seen in other specimens. In the 3 years before the audit and feedback strategy breast and colon tumors made up $\sim 70 \%$ of tumor specimens biobanked, and although these are the most common tumor resection specimens received in our department this was probably an overrepresentation of our true departmental case mix. These specimens now represent $44 \%$ of the total specimens sampled for the biobank over this period compared to $57 \%$ and $74 \%$ respectively in the two preceding years.

Implementation of the audit and feedback strategy was so effective that all three targeted specimens saw a statistically significant increase ( $p$ value $<0.001$ ) in tumors sampled by the anatomical pathology department over this period compared to the preceding period, confirming the effectiveness of this strategy in modifying tumor sampling behavior of anatomical pathology registrars (Table 1).

Although the initial implementation of the audit and feedback strategy had the most significant effect on tumor sampling, additional email reinforcement also played a significant role in tumor sampling. For example, the additional blocks gained from reinforcement emails demonstrated a further statistically significant increase in tumor sampled from brain and lymph node specimens when compared with samples taken at initial dissection, although not to the same degree of statistical significance $(p<0.05)$. The impact of this strategy on the department and people involved was minor, and although there was a predictable initial increase in the number of cases requiring feedback emails and biobanking after initial dissection, this reduces substantially as biobanking quickly becomes part of routine specimen dissection. The average time taken for each component of the audit and feedback strategy is illustrated in Figure 2, which is a small price to pay for such a significant outcome.

One drawback with this method is that notification of a potentially "biobankable" specimen that has not been sampled usually takes a minimum of 1 week to be reevaluated after the initial dissection due to administrative lag time. Therefore, these specimens spend at least 1 week in formalin before being sampled, resulting in extended formalin exposure. A record of cases with extended formalin exposure must be kept because these samples may be unsuitable for certain analytical tests. One could argue that this tissue should not be sampled due to the effect of overfixation on tissue antigenicity ${ }^{8}$ and DNA/RNA. However, the feedback component is a crucial part of this strategy, maintaining its effectiveness over a long period of time by keeping the initiative fresh in the minds of registrars and making it inconvenient not to evaluate the tumor for biobanking at initial dissection.
Among the three specimen types studied in detail, feedback emails occurred most frequently with lymph node excisions, with 22 out of 40 specimens sampled only after a feedback email to the operator responsible for the initial dissection.

This may be because lymph node excision specimens are often taken by for diagnosis and therefore the dissector does not know whether the specimen represents tumor tissue or reactive process and applies a cautious approach by not submitting the tissue for biobanking.

One solution to the problem of overfixation in the case of lymph node excisions would be to put any remaining tumor tissue into extra biobanking blocks at the time of initial dissection without sections cut for diagnosis. Once the case has been completed the blocks can then be biobanked, disposed of, or if diagnostically challenging, additional diagnostic sections can be cut without tissue being affected by overfixation, which would benefit additional diagnostic testing and biobanked tissue.

Although the results of the audit and feedback strategy have successfully increased the number of tumors sampled for the biobank, there is still room for improvement in terms of staff education and our methodology.

First, we missed several cases in each of the three specimen types (two brain, three lungs, and seven lymph nodes) that would have been amenable to biobanking as a result of administrative errors. In these cases, specimens were on the list of raw specimens to be checked, but were not transferred onto the final spreadsheet for feedback/email reinforcement by the pathologist. These cases may have been missed in error or the pathology report may have been misinterpreted as not being eligible for biobanking and therefore no "feedback" email was generated. This process is currently performed by technical officers so to improve this process, further training. Training of technical officers could be performed or this responsibility could be performed by an experienced anatomical pathology registrar.

Second, feedback emails were sent to registrars asking for tumor biobanking blocks on several cases deemed amenable to biobanking (six brain tumor specimens and four lymph node excisions) but no tumor blocks or other response was received. This may be because they were forgotten, they chose not to or the returning registrar did reevaluate the specimen but no tumor was available for sampling and this was not recorded in the in the LIS. This particular process could be improved by asking the registrar who performed the original specimen dissection to provide a confirmation email detailing whether a block had been taken, which would enable a biobank technical officer to issue a reminder whether it was forgotten or the registrar responsible was on leave.

Although the audit led to an increase in tumor biobanking across all specimens a small number of specimen types still demonstrated a low rate of biobanking due to specimen specific blocking protocols and/or preoperative management.

For example, patients undergoing esophageal, and to some extent rectal and bladder tumor resections commonly have a course of preoperative chemoradiotherapy that reduces the macroscopic tumor size for sampling. This in addition to comprehensive blocking protocols that require embedding of the entire tumor for diagnostic assessment makes these samples unsuitable for biobanking.

Biobanking of tumor samples from a radical prostatectomy is also difficult due to two factors. First, it is recommended that 
the entire prostate is embedded for assessment, therefore no "excess" tumor is available for biobanking, and second prostate carcinoma is difficult to identify macroscopically. Therefore, even if a small amount of "tumor tissue" is biobanked at initial dissection it may not be representative of the tumor.

The answer to the issues of small tumor size and embedding of the entire tumor may be to submit a thin slice of macroscopic tumor for biobanking that can be evaluated by the reporting pathologist, and if required for the case this block can then be converted back to a diagnostic block. In cases where a tumor may be difficult to evaluate macroscopically, such as prostate carcinoma, the results of previous biopsies can be used to guide biobanking blocks, which may improve sampling accuracy.

In summary, the introduction of an audit and feedback strategy is successful in modifying the sampling behavior of anatomical pathology registrars, as evidenced by increasing the overall number of tumor specimens sampled for the HCB by $141 \%$ compared with the previous period. The largest increases were seen in previously underrepresented specimens such as brain, lung, and lymph node tumor tissue, all of which have shown statistically significant increases in tumors sampled for the biobank during the study period. However, this success is tempered by small disadvantages in methodology with prolonged formalin fixation of tumor tissue retrieved after initial dissection and loss of specimens due to administrative error, which can be prevented or minimized in the future by changing tumor blocking protocol, implementation of specimen quality indicators and education of biobank personnel. A major benefit is obtained by having a dedicated biobank technician and a pathologist with a special interest in biobanking to implement the audit and feedback strategy.

\section{Acknowledgments}

Hunter Cancer Research Alliance (HCRA).

\section{Author Disclosure Statement}

No conflicting financial interests exist.

\section{References}

1. Organisation for Economic Co-operation and Development. Glossary of statistical terms. OECD Website. https://stats
.oecd.org/glossary/detail.asp?ID=7220. Published 2007. (Last accessed June 13, 2018).

2. National Pathology Accreditation Advisory Council. Requirements for the Performance of Anatomical Pathology Cut-Up (Fourth Edition, 2013). https://www.health.gov.au/ internet/main/publishing.nsf/Content/B2227CA579000C57 CA257BF0001E032D/\$File/V0.26\%20Anatomical\%20Cut-Up .pdf (accessed December 24, 2018).

3. Moran S, Vizoso M, Martinez-Cardús A, et al. Validation of DNA methylation profiling in formalin-fixed paraffin-embedded samples using the Infinium HumanMethylation450 Microarray. Epigenetics 2014;9:829-833.

4. Nasri S, Anjomshoaa A, Song S, et al. Oligonucleotide array outperforms SNP array on formalin-fixed paraffin-embedded clinical samples. Cancer Genet Cytogenet 2010;198:1-6.

5. Tramm T, Hennig G, Kyndi M, et al. Reliable PCR quantitation of estrogen, progesterone and ERBB2 receptor mRNA from formalin-fixed, paraffin-embedded tissue is independent of prior macro-dissection. Virchows Arch 2013; 463:775-786.

6. Ivers N, Jamtvedt G, Flottorp S, et al. Audit and feedback: effects on professional practice and healthcare outcomes. Cochrane Database of Systematic Reviews 2012, Issue 6. Art. No.: CD000259. DOI: 10.1002/14651858.CD000259.pub3.

7. Flottorp SA, Jamtvedt G, Gibis B, McKee M. Using Audit and Feedback to Health Professionals to Improve the Quality and Safety of Health Care. World Health Organization. 2010. www.euro.who.int/_data/assets/pdf_file/0003/124419/ e94296.pdf.

8. Hussong JW, Arber DA, Bradley KT, et al. Protocol for the examination of specimens from patients with non-Hodgkin lymphoma/lymphoid neoplasms. Arch Pathol Lab Med 2010; 134:e40-7. 